\title{
Electric circuits for universal quantum gates and quantum Fourier transformation
}

\author{
Motohiko Ezawa () \\ Department of Applied Physics, University of Tokyo, Hongo 7-3-1, 113-8656, Japan
}

(Received 6 November 2019; accepted 20 May 2020; published 3 June 2020)

\begin{abstract}
A set of universal quantum gates can be constructed based on quantum walk by formulating it as a scattering problem on a graph. In this paper, we simulate quantum gates by electric circuits, following a recent report that a one-dimensional $L C$ electric circuit can simulate a Schrödinger equation and hence a quantum walk. Especially, we propose a physical realization of a set of universal quantum gates consisting of the CNOT, Hadamard, and $\pi / 4$ phase-shift gates with the use of telegrapher wires and mixing bridges. Furthermore, we construct the $\pi / 2^{n}$ phase-shift gate for an arbitrary integer $n$, which is an essential element to perform the quantum Fourier transformation and prime factorization based on the Shor algorithm. Our results will open a way to simulate any quantum algorithms based on electric circuits.
\end{abstract}

DOI: 10.1103/PhysRevResearch.2.023278

\section{INTRODUCTION}

Quantum computation [1,2] is a most urgent and promising next-generation technique, which overcomes the limit of the Moore law. Universal quantum computation is indispensable to make any quantum algorithms possible [3]. According to the Solovay-Kitaev theorem, a set of the universal gates consists of two Clifford and one non-Clifford gates [4]. The standard set is given by the controlled-NOT (CNOT), Hadamard, and $\pi / 4$ phase-shift gates [5], where the $\pi / 4$ phase-shift gate is a non-Clifford gate. Any unitary transformation is executable with the use of these operators. One of the methods to realize universal quantum computation is based on quantum walks [6-15], where widgets act as quantum gates. During the past decades, there have been extensive investigations of quantum walks in a variety of physical platforms including $\mathrm{SiO}$ chips [16], superconducting qubits [17], and optical lattices $[18,19]$.

Recently, electric circuits attracted much attention in the context of topological physics [20-31]. Majoranalike topological edge states can be simulated in electric circuits [30] and topological quantum computation would be possible based on their braiding [32]. On the other hand, it has been shown [33] that quantum walks are simulated by the telegrapher circuit because the mathematical equivalence holds between the Kirchhof equation and the Schrödinger equation for the telegrapher circuit.

Feynman's original idea [1] is to use the Schrödinger equation for computations, since it describes natural phenomena. Indeed, the time evolution of a natural phenomenon is executed automatically at high speed. Our idea is similar to it, where we use the Kirchhof equation for computations.

Published by the American Physical Society under the terms of the Creative Commons Attribution 4.0 International license. Further distribution of this work must maintain attribution to the author(s) and the published article's title, journal citation, and DOI.
In this paper, we present a physical realization of a set of the CNOT, Hadamard, and $\pi / 4$ phase-shift gates with the use of $L C$ electric circuits. We consider a telegrapher circuit, which we map to a graph $G$ by identifying nodes and links as vertices and edges, respectively. We introduce a subdivided graph $G^{\prime}$ by transforming a link to a vertex. The graph $G^{\prime}$ is bipartite since it involves two types of vertices, representing voltage and current physically. The simplest example is the one-dimensional telegrapher wire illustrated in Fig. 1(a), which corresponds to graphs $G$ and $G^{\prime}$ in Figs. 1(b) and 1(c), respectively. Quantum walks on graph $G^{\prime}$ have already been explored [33]. We note that once a subdivided graph is given, the corresponding electric circuit is readily designed.

The next-simplest telegrapher circuit consists of four semiinfinite wires for two inputs and two outputs, connected to a certain finite circuit called a widget. It corresponds to a quantum gate represented by a $2 \times 2$ matrix. There are three building blocks, the mixing gate $U_{\text {mix }}^{(2)}$, the $\phi$ phase-shift gate $U_{\phi}$, and the NOT gate or the Pauli $X$ gate $U_{X} \equiv \sigma_{x}$, where

$$
\begin{aligned}
U_{\text {mix }}^{(2)} & =\frac{1}{\sqrt{2}}\left(\begin{array}{cc}
i & -1 \\
-1 & i
\end{array}\right), \quad U_{\phi}=\left(\begin{array}{cc}
1 & 0 \\
0 & e^{i \phi}
\end{array}\right), \\
U_{X} & =\left(\begin{array}{ll}
0 & 1 \\
1 & 0
\end{array}\right) .
\end{aligned}
$$

The mixing gate corresponds to the one named basis-changing gate in literature [6]. The phase-shift gate $U_{\phi}$ is constructed simply by inserting inductors due to the induced electromotive force. However, the mixing gate contains two bridges across two wires as in Fig. 2, and causes a back scattering of quantum walkers by the bridges in general. Note that the superscript (2) in $U_{\text {mix }}^{(2)}$ implies the number of the bridges. The suppression of back scattering is necessary for quantum gates, which restricts the momentum associated with a quantum walker. Possible values of the phase shift $\phi$ are determined by this condition. The phase shift $\phi=\pi / 4$ is such an allowed one, producing the $\pi / 4$ phase-shift gate as in Fig. 3. The Hadamard gate is constructed as in Fig. 4 in accord with the relation $U_{\mathrm{H}}=$ $-i U_{3 \pi / 2} U_{\text {mix }}^{(2)} U_{3 \pi / 2}$, where $U_{3 \pi / 2}=\left(U_{\pi / 4}\right)^{3}$. The NOT gate is 
(a) Telegrapher circuit

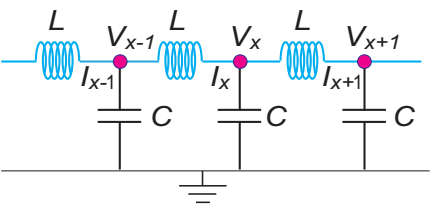

(b) Graph G

(c) Subdivided graph G'

FIG. 1. (a) Illustration of a one-dimensional telegrapher circuit, where two nodes (in magenta) are connected by inductor $L$ in cyan. Each node is connected to the ground by capacitor $C$. (b) Illustration of the associated graph $G$. (c) Illustration of the subdivided graph $G^{\prime}$, where cyan vertices correspond to inductors $L$.

simply given by exchanging two wires as in Fig. 5(a). The CNOT gate transforms four inputs to four outputs, whose main part is the NOT gate as in Fig. 5(c). The standard set of universal gates is constructed in this way.

A typical application of quantum computers is prime factorization. A key algorithm is the quantum Fourier transformation. It is decomposed into the Hadamard gate $U_{\mathrm{H}}$ and the $\pi / 2^{n}$ phase-shift gate with integer $n$ in Eqs. (1). Although any quantum gate may be constructed based on the standard set of universal gates, it is better to employ the $\pi / 2^{n}$ phase-shift gate directly for practical purposes. We are able to construct it together with the mixing gate $U_{\text {mix }}^{(N)}$ by introducing $N$ bridges across two wires with $N=2^{n-1}$ as in Fig. 6 .

Here we summarize the main differences between the previous studies [6-8] and the present one as graph theory. First, the graph is bipartite in the present scheme because it describes a network of voltage and current. Second, the hopping parameters are pure imaginary as in Eq. (6), as

(a) Mixing-gate circuit
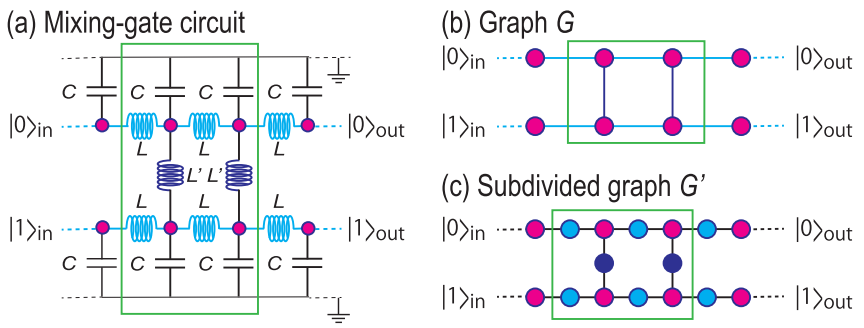

(c) Subdivided graph $G^{\prime}$

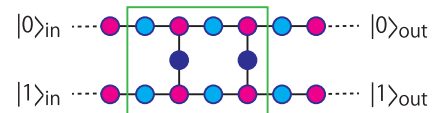

(d) Absolute value of $\mathrm{Tj}$ and $\mathrm{R}$

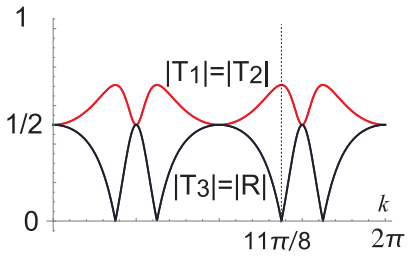

(e) Angle of $T_{j}$ and $R$

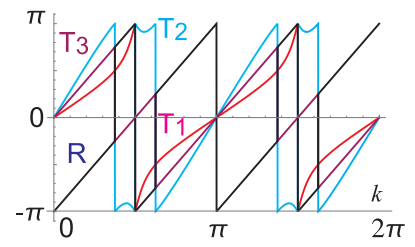

FIG. 2. (a) Illustration of an electric circuit realizing the mixing gate $U_{\text {mix }}^{(2)}$ marked by a green rectangle. It consists of two parallel wires linked by two inductors $L^{\prime}$. (b) Graph $G$ for the mixing gate. (c) The dynamics of the electric circuit is equivalent to a quantum walk on the subdivided graph $G^{\prime}$. (d), (e) The $k$ dependence of the absolute value and the phase of the transmission coefficients $T_{1}(k)$, $T_{2}(k), T_{3}(k)$, and the reflection coefficient $R(k) . T_{1}$ is colored in red, $T_{2}$ is colored in cyan, $T_{3}$ is colored in violet, and $R$ is colored in black. The horizontal axis is the momentum $0 \leqslant k \leqslant 2 \pi$. We find $\left|T_{1}(k)\right|=\left|T_{2}(k)\right|$ and $\left|T_{3}(k)\right|=|R(k)|$.

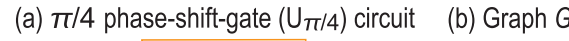

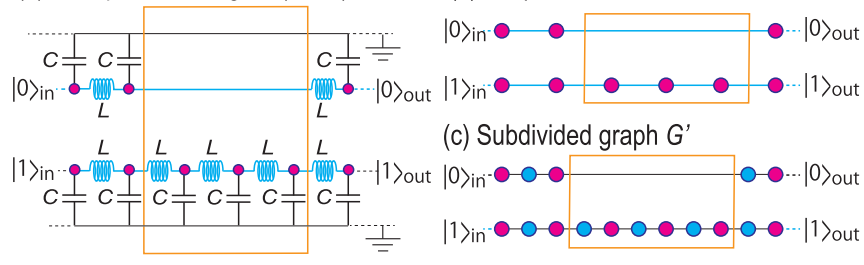

FIG. 3. (a) Illustration of an electric circuit for the $\pi / 4$ phaseshift gate. Within the widget, there are no electronic parts in the upper wire, while there are three inductors $L$ in the lower wire, producing the phase delay $2 m k_{0}$ with $m=3$ in the lower wire compared to the upper wire. (b), (c) Associated graphs.

originates in the mapping of the telegrapher equation to the Schrödinger equation. Third, the amplitude of the hopping is not uniform because we use two types of inductors $L$ and $L^{\prime}$ as in Fig. 2, which makes the graph weighted.

This paper is composed as follows. In Sec. II, we review how the Kirchhoff laws for the voltage and the current are rewritten in the form of the two-component Schrödinger equation on a graph, which allows us to consider one-dimensional quantum walk along telegrapher wires. In Sec. III, we analyze a scattering theory on a system made of two infinite telegrapher wires with a widget where the two wires are linked by two bridges. The condition that there is no back scattering determines the momentum associated with a quantum walker. In Sec. IV, we study one-qubit gates made of widgets involving two telegrapher wires. They include the phase-shift gate, the mixing gate, the Hadamard gate, and the Pauli gates. In Sec. V, we study two-qubit gates made of widgets involving four telegrapher wires. They include the CNOT gate, the SWAP gate, the controlled- $Z(\mathrm{CZ})$ gate, and the controlled phase-shift gate. In Sec. VI, we study three-qubit gates such as the Toffoli gate and the Fredkin gate. In Sec. VII, we investigate how to perform quantum Fourier transformation based on electric circuits. In Sec. VIII, we discuss how the quantum gate is robust against randomness in capacitors and inductors, and

\section{(a) Hadamard-gate circuit}

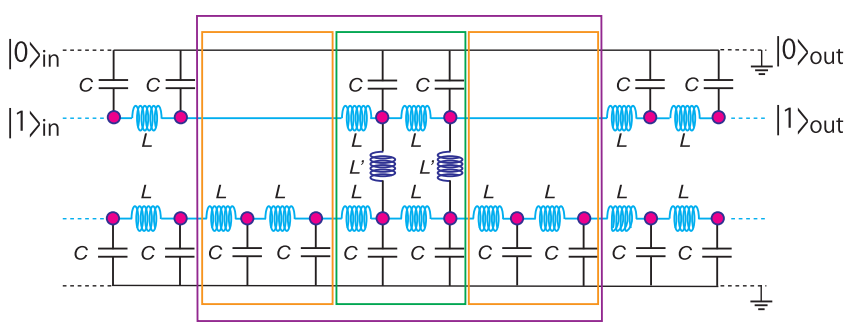

(b) Graph G' for Hadamard gate

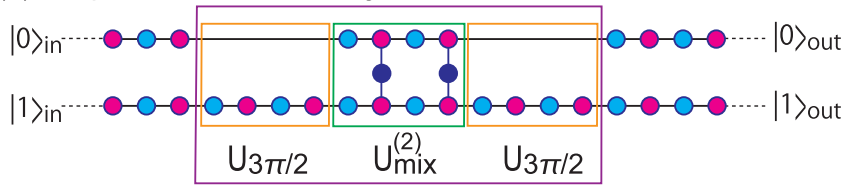

FIG. 4. (a) Illustration of an electric circuit for the Hadamard gate. It consists of the successive operations of the $3 \pi / 2$ phase shift and mixing gates. (b) Graph representation of the Hadamard gate. 


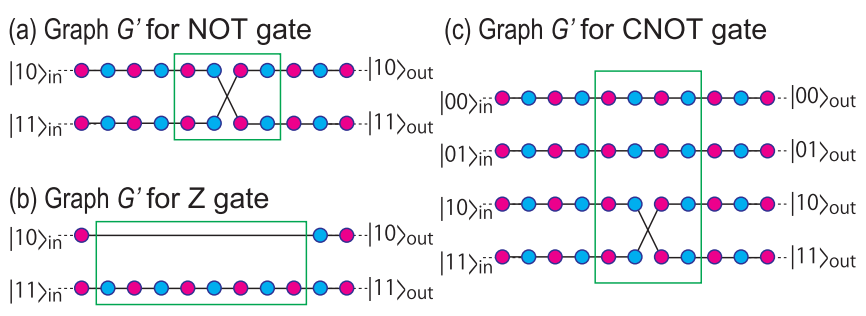

FIG. 5. (a)-(c) Graph representation of the Pauli $X$ gate, the Pauli $Z$ gate, and the CNOT gate.

also in the presence of resistance. Section IX is devoted to the conclusion.

\section{QUANTUM WALKS AND TELEGRAPHER EQUATION}

Our basic idea is to use telegrapher wires for a physical realization of quantum walks. A telegrapher wire is a playground for one-dimensional quantum walkers [33], where each node is connected to the ground by capacitor $C$, and an inductor $L$ is inserted to each link, as illustrated in Fig. 1. We also consider a widget connected to semi-infinite wires. For example, there are two input wires and two output wires connected to a widget marked in green in Fig. 2. The key problem is whether a quantum walker may get through it without reflection.

An $L C$ electric circuit is characterized by the Kirchhoff laws for voltage $V_{x}$ at node $x$ and current $I_{x}$ entering node $x$,

$$
\begin{aligned}
L_{x} \frac{d}{d t} I_{x}(t) & =V_{x^{\prime}}(t)-V_{x}(t), \\
C_{x} \frac{d}{d t} V_{x}(t) & =I_{x}(t)+\sum_{x^{\prime}} s I_{x^{\prime}}(t),
\end{aligned}
$$

where $x^{\prime}$ is one of the neighboring nodes of $x$ such that the current is $I_{x}$ between these two nodes. The sign $s= \pm$ is determined whether the current is ingoing or outgoing. The first equation is the Kirchhoff voltage law with respect to the voltage difference between two nodes $V_{x}$ and $V_{x^{\prime}}$, which originates in the induced electromotive force by the inductor $L_{x}$. The second equation is the Kirchhoff current law with respect to the current conservation at one node $V_{x}$. We denote a vertex representing the voltage $V_{x}$ (the current $I_{x}$ ) in magenta (cyan) in figures.

We may rewrite the Kirchhoff laws Eqs. (2) and (3) in the form of the Schrödinger equation on graph $G$,

$$
i \frac{d}{d t} \psi(x, t)=H \psi(x, t),
$$

(a) Graph $G^{\prime}$ for mixing gate $U_{\text {mix }}^{(M)}$

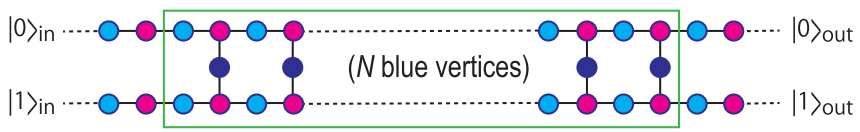

FIG. 6. (a) Graph $G^{\prime}$ representation of the mixing gate $U_{\text {mix }}^{(N)}$ with $N=2^{n-1}$, which is composed of two wires linked by $N$ inductors $L^{\prime}$ (in blue). It is used to construct the $\pi / 2^{n}$ phase-shift gate. as we have presented a concrete example for one-dimensional quantum walks elsewhere [33]. Here, the wave function is a two-component vector:

$$
\psi(x, t)=\left(\begin{array}{l}
\mathcal{I}_{x}(t) \\
\mathcal{V}_{x}(t)
\end{array}\right) \equiv\left(\begin{array}{c}
V_{x}(t) \\
\sqrt{L / C} I_{x}(t)
\end{array}\right) .
$$

The Hamiltonian $H$ is given by the Kirchhoff laws when an electric circuit is given.

We make separation of variables, $V_{x}(t)=\operatorname{Re}\left[V(x) e^{i \omega t}\right]$ and $I_{x}(t)=\operatorname{Re}\left[I(x) e^{i \omega t}\right]$, where $V(x)$ and $I(x)$ are complex voltage and current. Correspondingly, we define the complex function $\psi(x)$ by $\psi(x, t)=\operatorname{Re}\left[\psi(x) e^{i \omega t}\right]$.

The aim of this paper is to propose a set of widgets which act as a set of universal gates and also some other gates. The first step is to solve the Hamiltonian problem of an infinite one-dimensional wire, where the Hamiltonian is given by [33]

$$
H=\frac{2}{\sqrt{L C}} \sum_{x}(i|\psi(x)\rangle\langle\psi(x+1)|-i| \psi(x+1)\rangle\langle\psi(x)|) \text {. }
$$

In the momentum space, the Schrödinger Eq. (4) reads

$$
i \frac{d}{d t} \psi_{k}=H(k) \psi_{k}
$$

where

$$
H(k)=\left(\begin{array}{cc}
0 & -\frac{i}{\sqrt{L C}}\left(1-e^{-i k}\right) \\
\frac{i}{\sqrt{L C}}\left(1-e^{i k}\right) & 0
\end{array}\right) .
$$

The eigen-energy is obtained as $E(k)=(2 / \sqrt{L C}) \sin k$.

Then, we solve the Schrödinger Eq. (4) for a system where semi-infinite wires are connected to a widget [6]. The key point is to formulate the system as a scattering problem on a graph, where the wave function is given by solving the eigen-equation [6-8],

$$
H \psi_{k}(x)=(2 / \sqrt{L C}) \sin k \psi_{k}(x),
$$

by requiring that the eigenenergy is the same as that of the semi-infinite wire.

\section{SCATTERING THEORY ON GRAPHS}

We start with a one-qubit gate $U$ from the input $\left(|0\rangle_{\text {in }},|1\rangle_{\text {in }}\right)$ to the output $\left(|0\rangle_{\text {out }},|1\rangle_{\text {out }}\right)$ :

$$
\left(\begin{array}{c}
|0\rangle_{\text {out }} \\
|1\rangle_{\text {out }}
\end{array}\right)=U\left(\begin{array}{c}
|0\rangle_{\text {in }} \\
|1\rangle_{\text {in }}
\end{array}\right) \text {. }
$$

We realize it by a set of two telegrapher wires containing a finite number of widgets, $U=U_{1} U_{2} \cdots$. We first analyze a widget illustrated in Fig. 2(a) explicitly, where two telegrapher wires are bridged by two inductors $L^{\prime}$.

Linear electric circuits satisfy the superposition principle. Hence, it is enough to calculate the transmission and reflection coefficients when we input a plane wave only to the wire $|0\rangle_{\text {in }}$. There are three other lines, where two of them are the outputs and the rest is the other input. This is a scattering problem, and the wave functions are written in the form of

$$
\begin{aligned}
\langle x, 0 \mid \psi\rangle & =e^{-i k x}+R(k) e^{i(k+\pi) x}, \\
\langle x, j \mid \psi\rangle & =T_{j}(k) e^{i k x},
\end{aligned}
$$


where $T_{1}$ and $T_{2}$ are for the two outputs, while $T_{3}$ is for the other input $j=1,2,3$. The momentum of the reflected wave is $k+\pi$ to preserve the energy $E(k)$. To make a quantum gate, it is necessary to determine the momentum $k=k_{0}$ by requiring $R\left(k_{0}\right)=0$ and $T_{3}\left(k_{0}\right)=0$ since the current should not backflow to the two inputs.

By solving the eigenequation Eq. (9), we obtain

$$
\begin{aligned}
& T_{1}(k)=\frac{e^{-2 i k}+2 \ell+e^{2 i k}\left(2 \ell^{2}-1\right)}{e^{-4 i k}+e^{-2 i k} 4 \ell+\left(4 \ell^{2}-1\right)}, \\
& T_{2}(k)=\frac{2 \ell\left(e^{2 i k} \ell+1\right)}{e^{-4 i k}+4 e^{-2 i k} \ell+\left(4 \ell^{2}-1\right)}, \\
& T_{3}(k)=\frac{2 \ell(\ell+\cos 2 k)}{e^{-4 i k}+4 e^{-2 i k} \ell+\left(4 \ell^{2}-1\right)}, \\
& R(k)=\frac{-2 \ell(\ell+\cos 2 k)}{e^{-4 i k}+4 e^{-2 i k} \ell+\left(4 \ell^{2}-1\right)},
\end{aligned}
$$

where $\ell=L^{\prime} / L$. The reflection becomes zero, $R\left(k_{0}\right)=0$, at $k_{0} \equiv \pm \frac{1}{2} \arccos (-\ell)+\eta \pi$ with $\eta=0,1$. At this momentum $k_{0}$, it follows that $T_{3}\left(k_{0}\right)=0, T_{1}\left(k_{0}\right)= \pm i \sqrt{1-\ell^{2}}$ and $T_{2}\left(k_{0}\right)=-\ell$. Hence we obtain

$$
U_{\operatorname{mix}}^{(2) \pm}=\left(\begin{array}{cc} 
\pm i \sqrt{1-\ell^{2}} & -\ell \\
-\ell & \pm i \sqrt{1-\ell^{2}}
\end{array}\right)
$$

for a possible widget acting as a quantum gate.

The Hadamard gate $U_{\mathrm{H}}$ is defined by

$$
U_{\mathrm{H}} \equiv \frac{1}{\sqrt{2}}\left(\begin{array}{cc}
1 & 1 \\
1 & -1
\end{array}\right) \text {. }
$$

To construct it from $U_{\text {mix }}^{(2) \pm}$, we need to set $\left|T_{1}(k)\right|=\left|T_{2}(k)\right|$, which is achieved by choosing $\ell=1 / \sqrt{2}$. The quantum gate Eq. (17) reads

$$
U_{\operatorname{mix}}^{(2) \pm}=\frac{1}{\sqrt{2}}\left(\begin{array}{cc} 
\pm i & -1 \\
-1 & \pm i
\end{array}\right)
$$

which we call the mixing gate. This corresponds to the one named basis-changing gate in literature [6]. Here, $U_{\text {mix }}^{(2)+}$ is realized at momenta $k_{0}=3 \pi / 8,11 \pi / 8$, while $U_{\text {mix }}^{(2)-}$ is realized at momenta $k_{0}=5 \pi / 8,13 \pi / 8$.

We show the transmission and reflection coefficients as a function of $k$ for $\ell=1 / \sqrt{2}$ in Figs. 2(d) and 2(e). We find $\left|T_{1}(k)\right|=\left|T_{2}(k)\right|$ and $|R(k)|=\left|T_{3}(k)\right|$ for all $k$.

\section{ONE-QUBIT GATES}

\section{A. Phase-shift gate}

We construct a phase-shift gate. It is simply constructed by inserting $m$ inductors only in the lower wire compared to the upper wire as shown in Fig. 3. The transmission and reflection coefficients are given by

$$
T(k)=e^{2 i m k}, \quad R(k)=0 .
$$

The transmission is perfect irrespective of the momentum since $|T(k)|=1$. Consequently, we obtain the $\phi$ mixing-gate $U_{\phi}$ with $\phi=2 m k$,

$$
U_{2 m}=\left(\begin{array}{cc}
1 & 0 \\
0 & e^{i 2 m k}
\end{array}\right) .
$$

It is understood physically that inserted inductors cause delay in the lower wire.

Here the momentum for a phase-shift gate should be the same as that for the mixing gate in one circuit. Then, we need to choose one momentum from $k_{0}=3 \pi / 8,11 \pi / 8$, $5 \pi / 8,13 \pi / 8$ for one circuit. Hereafter, we choose $k_{0}=$ $11 \pi / 8$ since it requires the minimum number of inductors to construct the $\pi / 4$ phase-shift gate.

By using three inductors, i.e., $m=3$ and $k_{0}=11 \pi / 8$, we obtain the $\pi / 4$ phase-shift gate as

$$
U_{\pi / 4}=\left(\begin{array}{cc}
1 & 0 \\
0 & e^{i \pi / 4}
\end{array}\right) .
$$

In general, by inserting $m$ inductors in one wire, we may generate the phase shift as follows:

\begin{tabular}{|c|c|c|c|c|c|c|c|c|}
\hline$m$ & 1 & 2 & 3 & 4 & 5 & 6 & 7 & 8 \\
\hline phase shift & $\frac{3 \pi}{4}$ & $\frac{3 \pi}{2}$ & $\frac{\pi}{4}$ & $\pi$ & $\frac{7 \pi}{4}$ & $\frac{\pi}{2}$ & $\frac{5 \pi}{4}$ & 0 \\
\hline
\end{tabular}

Two successive operations of the $\pi / 4$ phase-shift gate yield

$$
U_{\pi / 2}=U_{\pi / 4}^{2}=\left(\begin{array}{ll}
1 & 0 \\
0 & i
\end{array}\right),
$$

which is simply called the phase gate.

\section{B. Hadmard gate}

The Hadamard gate is the unitary operation $U_{\mathrm{H}}$ defined by Eq. (18). It is given by the combination of the mixing and $3 \pi / 2$ phase-shift gates as

$$
U_{\mathrm{H}}=-i U_{3 \pi / 2} U_{\text {mix }} U_{3 \pi / 2},
$$

where the $3 \pi / 2$ phase-shift gate is constructed by inserting two inductors sequentially. We show the graph $G^{\prime}$ for the Hadamard gate in Fig. 4.

\section{Pauli gates}

We study the NOT gate. It is defined by the interchange of two inputs $|0\rangle_{\text {in }}$ and $|1\rangle_{\text {in }}$ or

$$
|1\rangle_{\text {out }}=U_{X}|0\rangle_{\text {in }} \quad \text { and } \quad|0\rangle_{\text {out }}=U_{X}|1\rangle_{\text {in }} .
$$

Such a gate is obviously given by the Pauli $X$ gate, $U_{X}=\sigma_{x}$. In terms of electric circuits, two telegrapher wires are simply interchanged as shown in Fig. 5(a).

The Pauli $Z$ gate reads $U_{Z}=\sigma_{z}$ by choosing $m=4$ in Eq. (23). It is constructed by inserting four successive inductors only for the lower wire as shown in Fig. 5(b). The Pauli $Y$ gate reads $U_{Y}=i U_{X} U_{Z}=\sigma_{y}$, which is constructed by the successive operations of the Pauli $Z$ and $X$ gates.

\section{Square root of NOT gate}

The square roof of the NOT gate is given by

$$
U_{\sqrt{X}} \equiv \frac{1}{2}\left(\begin{array}{cc}
1+i & 1-i \\
1-i & 1+i
\end{array}\right),
$$

which is realized by the sequential application of the NOT gate and the mixing gates as

$$
U_{\sqrt{X}}=-e^{i \pi / 4} U_{\mathrm{mix}}^{(2)} U_{X} .
$$

It is easy to check $\left(U_{\sqrt{X}}\right)^{2}=U_{X}$. 


\section{E. $\pi / 2^{n}$ phase-shift gate}

We now construct the $\pi / 2^{n}$ phase-shift gate for an arbitrary integer $n$. First, we construct a mixing gate which matches with the $\pi / 2^{n}$ phase-shift gate, by generalizing the result for the $\pi / 4$ phase-shift gate. We bridge two telegrapher wires by $N \equiv 2^{n-1}$ inductors with inductance $L^{\prime}$, as illustrated in Fig. 6. The momentum $k_{0}$ and the inductance $L^{\prime}=\ell L$ should be determined so there are no reflections to the inputs. The condition is satisfied when we choose $k_{0}=5 \pi / 4+\pi /(4 N)$ and $\ell=-\cos 2 k_{0}$, where the system acts as the quantum gate given by

$$
U_{\mathrm{mix}}^{(N)}=\frac{e^{\frac{i \pi}{2} N}}{\sqrt{2}}\left(\begin{array}{cc}
\exp \left[-\frac{i \pi}{2}\left(\frac{1}{2}+\frac{1}{N}\right)\right] & \exp \left[\frac{i \pi}{2}\left(\frac{1}{2}-\frac{1}{N}\right)\right] \\
\exp \left[\frac{i \pi}{2}\left(\frac{1}{2}-\frac{1}{N}\right)\right] & \exp \left[-\frac{i \pi}{2}\left(\frac{1}{2}+\frac{1}{N}\right)\right]
\end{array}\right) .
$$

It is reduced to $U_{\text {mix }}^{(2)}$ in Eqs. (1) for $n=2$ or $N=2$, where $k_{0}=11 \pi / 8$ and $\ell=-\cos 11 \pi / 4=1 / \sqrt{2}$.

The $\pi / 2^{n}$ phase-shift gate is constructed by inserting $m$ inductors only in the lower wire as for the case of the $\pi / 4$ phase shift gate, yielding $U_{\phi}$ in Eqs. (1) with $\phi=2 m k_{0}$, where $m$ is determined by the condition $2 m k_{0}=\pi / 2^{n} \bmod (2 \pi)$.

The Hadamard gate is constructed so as to realize the mathematical relation,

$$
\exp \left[\frac{i \pi}{2}\left(\frac{1}{2}+\frac{1}{N}+N\right)\right] U_{3 \pi / 2} U_{\text {mix }}^{(N)} U_{3 \pi / 2}=U_{\mathrm{H}},
$$

where the $3 \pi / 2$ phase-shift gate $U_{3 \pi / 2}$ is constructed from the $\pi / 2^{n}$ phase-shift gate as $U_{3 \pi / 2}=\left(U_{\pi / 2^{n}}\right)^{3 N}$.

In general, we may construct the $\phi$ phase-shift gate $U_{\phi}$ for arbitrary $\phi$ from the $\pi / 2^{n}$ phase-shift gate as $U_{\phi}=\left(U_{\pi / 2^{n}}\right)^{s}$ with a certain integer $s$. It is well known that the relation $s / N=2 \phi / \pi$ holds within a required accuracy by taking two integers $s$ and $N$ appropriately.

\section{F. One-qubit universal gate}

We may construct a combination of the Hadamard and phase-shift gates such as

$$
U_{1 \mathrm{bit}}=e^{-i \theta / 2} U_{\phi+\pi} U_{\mathrm{H}} U_{\theta} U_{\mathrm{H}}=\left(\begin{array}{cc}
\cos \frac{\theta}{2} & -i \sin \frac{\theta}{2} \\
i e^{i \phi} \sin \frac{\theta}{2} & -e^{i \phi} \cos \frac{\theta}{2}
\end{array}\right) \text {, }
$$

which represents any $\mathrm{SU}(2)$ generator. It is called the onequbit universal quantum gate.

\section{TWO-QUBIT GATES}

\section{A. CNOT gate}

The CNOT gate is a two-qubit gate $U$, transforming the input $\left(|00\rangle_{\text {in }},|01\rangle_{\text {in }},|10\rangle_{\text {in }},|11\rangle_{\text {in }}\right)$ to the output $\left(|00\rangle_{\text {out }},|01\rangle_{\text {out }},|10\rangle_{\text {out }},|11\rangle_{\text {out }}\right)$. It is defined by

$$
U_{\mathrm{CNOT}} \equiv\left(\begin{array}{cc}
I_{2} & O_{2} \\
O_{2} & U_{X}
\end{array}\right)
$$

where $I_{2}$ is the two-dimensional identity matrix, $\mathrm{O}_{2}$ is the two-dimensional null matrix, and $U_{X}$ is the NOT gate. Its construction is straightforward. We show the graph for the CNOT gate in Fig. 5(c).

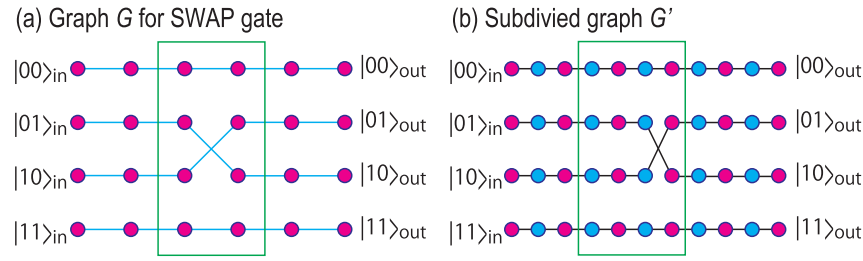

FIG. 7. Graph representation of the SWAP gate, where the $|01\rangle$ and $|10\rangle$ are interchanged.

\section{B. SWAP gate}

The SWAP gate is defined by

$$
U_{\text {SWAP }} \equiv\left(\begin{array}{cccc}
1 & 0 & 0 & 0 \\
0 & 0 & 1 & 0 \\
0 & 1 & 0 & 0 \\
0 & 0 & 0 & 1
\end{array}\right)
$$

It is constructed by the exchange of the wires between $|01\rangle_{\text {in }}$ and $|10\rangle_{\text {in }}$, which are shown in Fig. 7. The SWAP gate exchanges two qubits as $U_{\text {SWAP }}\left|j_{2} j_{1}\right\rangle=\left|j_{1} j_{2}\right\rangle$.

\section{CZ gate}

The CZ gate is defined by

$$
U_{\mathrm{CZ}} \equiv\left(\begin{array}{cc}
I_{2} & O_{2} \\
O_{2} & U_{\mathrm{Z}}
\end{array}\right)=\left(\begin{array}{cccc}
1 & 0 & 0 & 0 \\
0 & 1 & 0 & 0 \\
0 & 0 & 1 & 0 \\
0 & 0 & 0 & -1
\end{array}\right)
$$

We realize it by inserting four inductors only for the wire representing $|11\rangle_{\text {out }}$ as in Fig. 8.

\section{Controlled phase-shift gate}

The controlled phase shift gate $U_{2 \rightarrow \phi}$ is defined by

$$
U_{2 \rightarrow \phi} \equiv\left(\begin{array}{cc}
I_{2} & O_{2} \\
O_{2} & U_{\phi}
\end{array}\right)=\left(\begin{array}{cccc}
1 & 0 & 0 & 0 \\
0 & 1 & 0 & 0 \\
0 & 0 & 1 & 0 \\
0 & 0 & 0 & e^{i \phi}
\end{array}\right)
$$

We realize it by inserting an appropriate number of inductors only to the wire representing $|11\rangle_{\text {out }}$.

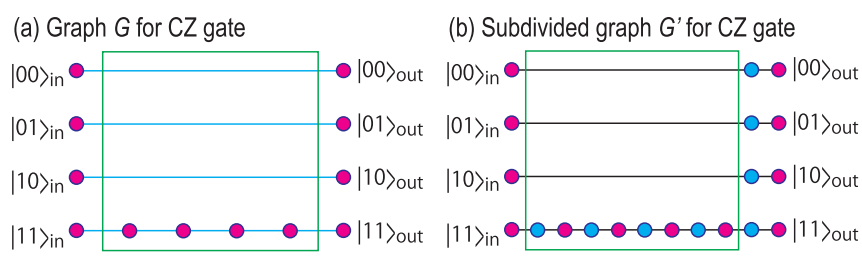

FIG. 8. Graph representation on the $\mathrm{CZ}$ gate. It consists of the operation of the $\pi$ phase-shift gate only for $|11\rangle$. 
(a) Graph G for Toffoli gate

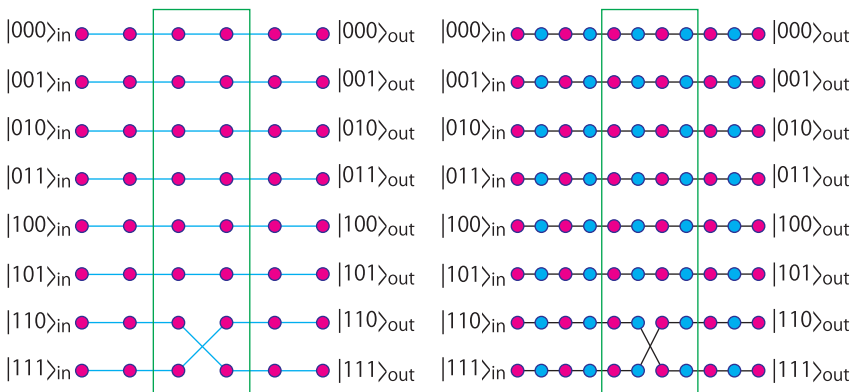

(c) Graph G for Fredkin gate

(d) Subdivided graph G' for Fredkin gate

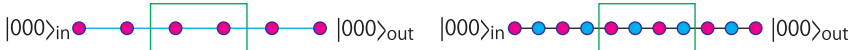

$\left.|001\rangle_{\text {in }} \bullet \bullet \quad \longrightarrow 001\right\rangle_{\text {out }}|001\rangle_{\text {in }} \bullet-0-0-0-0-0-0-0|001\rangle_{\text {out }}$

$|010\rangle_{\text {in }} \bullet|010\rangle_{\text {out }}|010\rangle_{\text {in }} \bullet-0-0-0-0-0-0-0|010\rangle_{\text {out }}$

$|011\rangle_{\text {in }}^{\longrightarrow} \bullet|011\rangle_{\text {out }}|011\rangle_{\text {in }} \bullet-0-0-0-0-0-0-0-0|011\rangle_{\text {out }}$

$|100\rangle_{\text {in }} \bullet \bullet \quad \bullet|100\rangle_{\text {out }}|100\rangle_{\text {in }} \bullet-0-0-0-0-0-0-0-0|100\rangle_{\text {out }}$

$\left.|101\rangle_{\text {in }} \bullet \bullet \bullet \cdot 101\right\rangle_{\text {out }}|101\rangle_{\text {in }} \bullet-0-0-0-0-0-0|101\rangle_{\text {out }}$

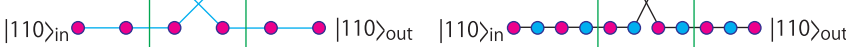

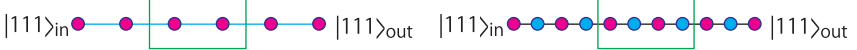

FIG. 9. Graph representations of the (a) Toffoli and (b) Fredkin gates.

\section{THREE-QUBIT GATES}

\section{A. Toffoli gate}

The Toffoli gate is a controlled-controlled NOT gate defined by

$$
\begin{aligned}
U_{\text {Toffoli }} \equiv & \left(\begin{array}{ccc}
I_{4} & O_{4} \\
O_{4} & U_{\mathrm{CNOT}}
\end{array}\right) \\
& =\left(\begin{array}{lllllllll}
1 & 0 & 0 & 0 & 0 & 0 & 0 & 0 \\
0 & 1 & 0 & 0 & 0 & 0 & 0 & 0 \\
0 & 0 & 1 & 0 & 0 & 0 & 0 & 0 \\
0 & 0 & 0 & 1 & 0 & 0 & 0 & 0 \\
0 & 0 & 0 & 0 & 1 & 0 & 0 & 0 \\
0 & 0 & 0 & 0 & 0 & 1 & 0 & 0 \\
0 & 0 & 0 & 0 & 0 & 0 & 0 & 1 \\
0 & 0 & 0 & 0 & 0 & 0 & 1 & 0
\end{array}\right),
\end{aligned}
$$

where $I_{4}$ is the four-dimensional identity matrix and $\mathrm{O}_{4}$ is the four-dimensional null matrix with the three-qubit basis $\{|000\rangle,|001\rangle,|010\rangle,|011\rangle,|100\rangle,|101\rangle,|110\rangle,|111\rangle\}^{t}$. We have

$$
\left(\begin{array}{l}
|000\rangle_{\text {out }} \\
|001\rangle_{\text {out }} \\
|010\rangle_{\text {out }} \\
|011\rangle_{\text {out }} \\
|100\rangle_{\text {out }} \\
|101\rangle_{\text {out }} \\
|110\rangle_{\text {out }} \\
|111\rangle_{\text {out }}
\end{array}\right)=U_{\text {Toffoli }}\left(\begin{array}{l}
|000\rangle_{\text {in }} \\
|001\rangle_{\text {in }} \\
|010\rangle_{\text {in }} \\
|011\rangle_{\text {in }} \\
|100\rangle_{\text {in }} \\
|101\rangle_{\text {in }} \\
|110\rangle_{\text {in }} \\
|111\rangle_{\text {in }}
\end{array}\right) .
$$

We use eight wires for three-qubit circuits. The two wires $|110\rangle_{\text {in }}$ and $|111\rangle_{\text {in }}$ are interchanged within the widget, while the others are directly connected to the outputs as shown in Fig. 9(a).

\section{B. Fredkin gate}

The Fredkin gate is a controlled SWAP gate defined by

$$
\begin{aligned}
U_{\text {Fredkin }} \equiv & \left(\begin{array}{ccc}
I_{4} & O_{4} \\
O_{4} & U_{\text {SWAP }}
\end{array}\right) \\
& =\left(\begin{array}{lllllllll}
1 & 0 & 0 & 0 & 0 & 0 & 0 & 0 \\
0 & 1 & 0 & 0 & 0 & 0 & 0 & 0 \\
0 & 0 & 1 & 0 & 0 & 0 & 0 & 0 \\
0 & 0 & 0 & 1 & 0 & 0 & 0 & 0 \\
0 & 0 & 0 & 0 & 1 & 0 & 0 & 0 \\
0 & 0 & 0 & 0 & 0 & 0 & 1 & 0 \\
0 & 0 & 0 & 0 & 0 & 1 & 0 & 0 \\
0 & 0 & 0 & 0 & 0 & 0 & 0 & 1
\end{array}\right) .
\end{aligned}
$$

Similarly to the Toffoli gate, the two wires $|101\rangle_{\text {in }}$ and $|110\rangle_{\text {in }}$ are interchanged within the widget while the others are directly connected to the outputs as shown in Fig. 9(b). Various arithmetic operations can be made by combinations of the NOT, CNOT, and Toffoli gates [34].

\section{QUANTUM FOURIER TRANSFORMATION}

The quantum Fourier transformation is defined by [35]

$$
|k\rangle=\frac{1}{\sqrt{N}} \sum_{j=0}^{N-1} \omega_{N}^{j k}|j\rangle
$$

with $\omega_{N}=e^{2 \pi i / N}$. It is constructed by successive operations of the Hadamard and $2 \pi / N$ phase-shift gates [36]. Thus, we are able to perform quantum Fourier transformation with arbitrary $N$. The quantum circuit representation of the quantum Fourier transformation is given in Fig. 10(a).

\section{A. Quantum Fourier transformation with $N=4$}

The quantum Fourier transformation for $N=4$ is explicitly given by

$$
U_{\mathrm{QFT} 2}=\frac{1}{2}\left(\begin{array}{cccc}
1 & 1 & 1 & 1 \\
1 & i & -1 & -i \\
1 & -1 & 1 & -1 \\
1 & -i & -1 & i
\end{array}\right) .
$$

It is decomposed into the sequential applications of the SWAP gate, the Hadamard gate, and the controlled-phase-shift gate $U_{2 \rightarrow \phi}$ as

$$
U_{\mathrm{QFT} 2}=\left(U_{\mathrm{H}} \otimes I_{2}\right)\left(U_{2 \rightarrow \pi / 2}\right)\left(I_{2} \otimes U_{\mathrm{H}}\right) U_{\mathrm{SWAP}},
$$

where

$$
\begin{aligned}
I_{2} \otimes U_{\mathrm{H}} & =\frac{1}{\sqrt{2}}\left(\begin{array}{cccc}
1 & 1 & 0 & 0 \\
1 & -1 & 0 & 0 \\
0 & 0 & 1 & 1 \\
0 & 0 & 1 & -1
\end{array}\right), \\
U_{\mathrm{H}} \otimes I_{2} & =\frac{1}{\sqrt{2}}\left(\begin{array}{cccc}
1 & 0 & 1 & 0 \\
0 & 1 & 0 & 1 \\
1 & 0 & -1 & 0 \\
0 & 1 & 0 & -1
\end{array}\right),
\end{aligned}
$$


(a)

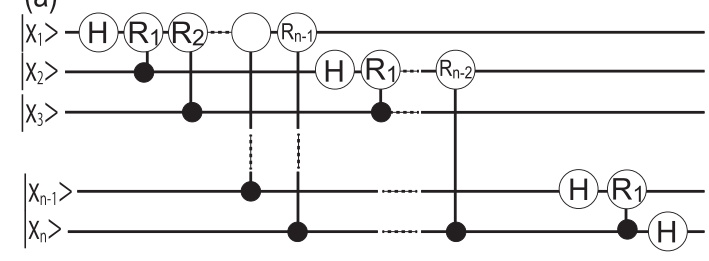

(b)
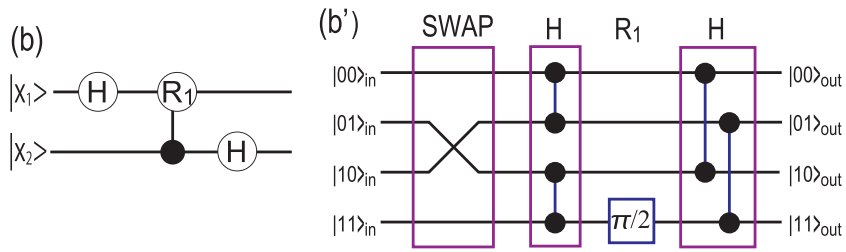

(c')
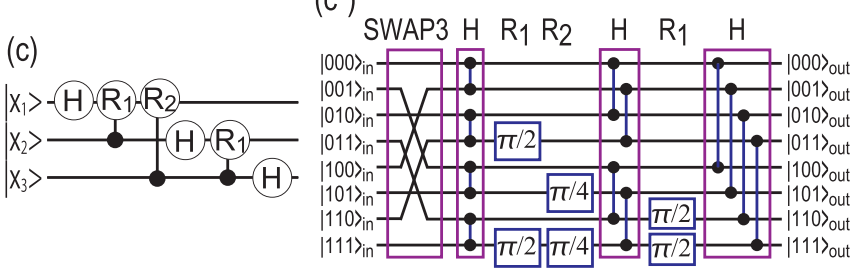

FIG. 10. (a) Quantum circuit representation of quantum Fourier transformation. The symbol $R_{n}$ indicates the $\pi / 2^{n}$ phase-shift gate while $H$ indicates the Hadamard gate. (b) Two-qubit quantum Fourier transformation. (c) Three-qubit quantum Fourier transformation. (b'), (c') The corresponding reduced graph representation.

$$
U_{2 \rightarrow \pi / 2}=\left(\begin{array}{cccc}
1 & 0 & 0 & 0 \\
0 & 1 & 0 & 0 \\
0 & 0 & 1 & 0 \\
0 & 0 & 0 & i
\end{array}\right) .
$$

The quantum circuit representation is given in Fig. 10(b). We have introduced the corresponding representation in terms of a reduced graph in Fig. 10( $\left.b^{\prime}\right)$, where each horizontal line stands for a telegrapher wire illustrated in Fig. 1(a); the SWAP gate stands for the interchange of two telegrapher wires as in Fig. 7; the $\mathrm{H}$ gate consists of some links connecting two wires, where each link stands for the Hadamard gate in Fig. 4; the $\mathrm{R}_{1}$ gate stands for the $\pi / 2$ phase-shift gate Eq. (24) obtained by two successive operations of the $\pi / 4$ phase-shift gate in Fig. 3 .

\section{B. Quantum Fourier transformation with $N=8$}

The quantum Fourier transformation for $N=8$ is explicitly given by

$$
U_{\mathrm{QFT} 3}=\frac{1}{2 \sqrt{2}}\left(\begin{array}{cccccccc}
1 & 1 & 1 & 1 & 1 & 1 & 1 & 1 \\
1 & \omega & \omega^{2} & \omega^{3} & \omega^{4} & \omega^{5} & \omega^{6} & \omega^{7} \\
1 & \omega^{2} & \omega^{4} & \omega^{6} & 1 & \omega^{2} & \omega^{4} & \omega^{6} \\
1 & \omega^{3} & \omega^{6} & \omega & \omega^{4} & \omega^{7} & \omega^{2} & \omega^{5} \\
1 & \omega^{4} & 1 & \omega^{4} & 1 & \omega^{4} & 1 & \omega^{4} \\
1 & \omega^{5} & \omega^{2} & \omega^{7} & \omega^{4} & \omega & \omega^{6} & \omega^{3} \\
1 & \omega^{6} & \omega^{4} & \omega^{2} & 1 & \omega^{6} & \omega^{4} & \omega^{2} \\
1 & \omega^{7} & \omega^{6} & \omega^{5} & \omega^{4} & \omega^{3} & \omega^{2} & \omega
\end{array}\right),
$$

with $\omega=e^{i \pi / 4}$.

It is decomposed into the sequential applications of the three-qubit SWAP gate $U_{\mathrm{SWAP} 3}$, the Hadamard gate, and the controlled-phase-shift gate $U_{n \rightarrow \phi}$ as

$$
\begin{aligned}
U_{\mathrm{QFT} 3}= & \left(U_{\mathrm{H}} \otimes I_{2} \otimes I_{2}\right)\left(U_{3 \rightarrow \pi / 2}\right)\left(I_{2} \otimes U_{\mathrm{H}} \otimes I_{2}\right) \\
& \times\left(U_{3 \rightarrow \pi / 4}\right)\left(U_{2 \rightarrow \pi / 2}\right)\left(I_{2} \otimes I_{2} \otimes U_{\mathrm{H}}\right) U_{\mathrm{SWAP} 3},
\end{aligned}
$$

with

$$
\begin{aligned}
& U_{2 \rightarrow \pi / 2}=\text { diag. }[1,1,1, i, 1,1,1, i], \\
& U_{3 \rightarrow \pi / 4}=\text { diag. }\left[1,1,1,1,1, e^{i \pi / 4}, 1, e^{i \pi / 4}\right], \\
& U_{3 \rightarrow \pi / 2}=\text { diag. }[1,1,1,1,1,1, i, i],
\end{aligned}
$$

and

$$
U_{\mathrm{SWAP} 3}\left|j_{3} j_{2} j_{1}\right\rangle=\left|j_{1} j_{2} j_{3}\right\rangle,
$$

with

$$
U_{\text {SWAP3 }}=\left(\begin{array}{cccccccc}
1 & 0 & 0 & 0 & 0 & 0 & 0 & 0 \\
0 & 0 & 0 & 0 & 1 & 0 & 0 & 0 \\
0 & 0 & 1 & 0 & 0 & 0 & 0 & 0 \\
0 & 0 & 0 & 0 & 0 & 0 & 1 & 0 \\
0 & 1 & 0 & 0 & 0 & 0 & 0 & 0 \\
0 & 0 & 0 & 0 & 0 & 1 & 0 & 0 \\
0 & 0 & 0 & 1 & 0 & 0 & 0 & 0 \\
0 & 0 & 0 & 0 & 0 & 0 & 0 & 1
\end{array}\right) .
$$

The quantum circuit representation is given in Fig. 10(c). The corresponding representation in terms of a reduced graph is given in Fig. 10 $\left(\mathrm{c}^{\prime}\right)$, which is explained similar to the case of the quantum Fourier transformation with $N=4$. New symbols in Fig. 10( $\left.c^{\prime}\right)$ are the SWAP3 gate representing the three-qubit swap Eq. (51), which is realized by interchanging telegrapher wires as indicated, and the $\mathrm{R}_{2}$ gate, standing for the $\pi / 4$ phase-shift gate in Fig. 3 .

\section{FIDELITY}

\section{A. Effects due randomness}

We study how the quantum gate is robust against randomness in capacitors and inductors. We introduce randomness into capacitors and inductors uniformly distributing from $-\delta$ to $\delta$ into a widget,

$$
C \longmapsto C(1+\eta \delta), \quad L \longmapsto L(1+\eta \delta),
$$

where $\eta$ is a random variable ranging from -1 to 1 . The robustness of the quantum gates is measured by the fidelity defined by

$$
F(|\phi\rangle,|\psi\rangle) \equiv|\langle\phi \mid \psi\rangle|,
$$

where $|\psi\rangle$ is the output from the ideal quantum gate while $|\phi\rangle$ is the output from the disordered quantum gate. The fidelity satisfies

$$
0 \leqslant F(|\phi\rangle,|\psi\rangle) \leqslant 1,
$$

where $F(|\phi\rangle,|\psi\rangle)=1$ dictates that the quantum gate is perfect.

We show the fidelity for the mixing gate as a function of the disorder $\delta$ in Fig. 11(a). It decreases as the disorder increases. On the other hand, the norm for the output from the disorderd quantum gate decreases as in Fig. 11(b). The norm is not equal to one since there is a reflection by the disordered gate. By comparing Figs. 11(a) and 11(b), it is found that the main 
reason for the decrease of the fidelity is due to the loss of the normalization. In Fig. 11(c), we show the fidelity for the normalized output from the disordered quantum gate defined by

$$
F_{N}\left(\frac{|\phi\rangle}{\| \phi\rangle \mid},|\psi\rangle\right) \equiv \frac{|\langle\phi \mid \psi\rangle|}{|| \phi\rangle \mid} .
$$

The fidelity remains close to one once the output is normalized. We show typical norm, fidelity, and normalized fidelity in a table as follows.

\begin{tabular}{lcccc} 
Sample disorder & $1 \%$ & $5 \%$ & $10 \%$ & $20 \%$ \\
\hline Norm & $0.9998 \pm 0.0002$ & $0.994 \pm 0.005$ & $0.976 \pm 0.018$ & $0.915 \pm 0.063$ \\
Fidelity $F(|\phi\rangle,|\psi\rangle)$ & $0.9997 \pm 0.0002$ & $0.992 \pm 0.005$ & $0.969 \pm 0.021$ & $0.887 \pm 0.071$ \\
Normalized Fidelity $F_{N}\left(\frac{|\phi\rangle}{\| \phi\rangle},|\psi\rangle\right)$ & $0.99993 \pm 0.00007$ & $0.998 \pm 0.002$ & $0.993 \pm 0.007$ & $0.972 \pm 0.029$
\end{tabular}

It is concluded that a very good fidelity is assured for the quantum gate provided the sample disorder is $1 \%$ or $5 \%$.

\section{B. Effects due to resistance}

We have so far neglected electric resistance to reveal the essential structure of quantum gates made of electric circuits. Electric resistance will also decrease the fidelity.

For a one-dimensional wire, the Kirchhoff law is modified as

$$
\begin{aligned}
L_{x} \frac{d}{d t} I_{x} & =V_{x-1}-V_{x}-R^{L} I_{x}, \\
C_{x} \frac{d}{d t} V_{x} & =I_{x}-I_{x+1}-V_{x} / R^{C},
\end{aligned}
$$

in the presence of parasite resistance $R^{L}$ and $R^{C}$ in inductors and capacitors. The Hamiltonian Eq. (8) becomes nonHermitian,

$$
H(k)=\left(\begin{array}{cc}
-i \frac{R^{L}}{L} & -\frac{i}{\sqrt{L C}}\left(1-e^{-i k}\right) \\
\frac{i}{\sqrt{L C}}\left(1-e^{i k}\right) & -i \frac{1}{C R^{C}}
\end{array}\right)
$$

For simplicity, we study the case $R^{L} / L=1 / C R^{C} \equiv R / \sqrt{L C}$, where we have introduced a dimensionless resistance $R$. Then, (a) 1.0

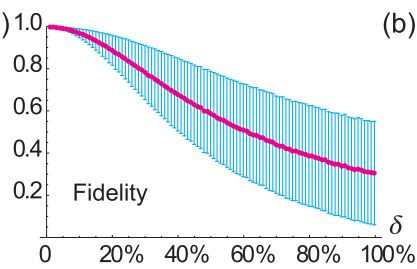

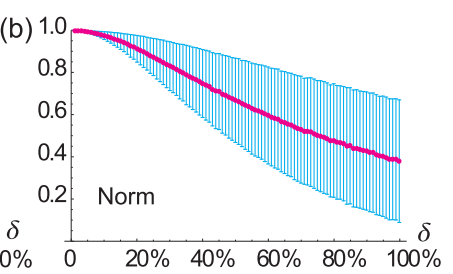

(c)

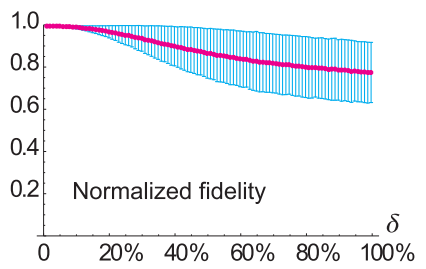

FIG. 11. (a) Fidelity $F(|\phi\rangle,|\psi\rangle)$. (b) Norm of the output from the disordered quantum gate. (c) Normalized fidelity $F_{N}\left(\frac{|\phi\rangle}{|\phi\rangle \mid},|\psi\rangle\right)$. The horizontal axis is the disorder strength $\delta$. The mean value is marked by a magenta dot while the standard deviation is plotted by a cyan error bar at each $\delta$. The average is taken 10000 times. the Hamiltonian is rewritten as

$$
H(k)=\frac{1}{\sqrt{L C}}\left(\begin{array}{cc}
-i R & -i\left(1-e^{-i k}\right) \\
i\left(1-e^{i k}\right) & -i R
\end{array}\right) .
$$

We show the fidelity for the mixing gate as a function of the resistance $R$ in Fig. 12(a). It decreases as the resistance increases, where the dominant contribution is the loss of the normalization. We show $\log _{10} F(|\phi\rangle,|\psi\rangle)$ in Fig. 12(b), where we find almost a linear relation. From this relation, we have an approximate relation:

$$
F(|\phi\rangle,|\psi\rangle) \simeq 10^{-R} .
$$

On the other hand, the normalized fidelity remains to be almost one, even for large resistance as in the case of the disorder in inductors and capacitors.

\begin{tabular}{lcccc} 
Resistance & $1 \%$ & $5 \%$ & $10 \%$ & $20 \%$ \\
\hline Norm & 0.962 & 0.830 & 0.700 & 0.516 \\
Fidelity $F(|\phi\rangle,|\psi\rangle)$ & 0.962 & 0.830 & 0.699 & 0.514 \\
Normalized Fidelity & 1 & 0.99997 & 0.9996 & 0.9966 \\
$10^{-R}$ & 0.977 & 0.891 & 0.794 & 0.631
\end{tabular}

\section{DISCUSSIONS}

We have presented a realistic proposal and systematic analysis of universal gate operations by simulating quantum walks with the use of electric circuits. However, the present scheme is not scalable. Namely, we need $2^{N}$ number of wires to realize $N$ qubits. Nevertheless, our approach has several
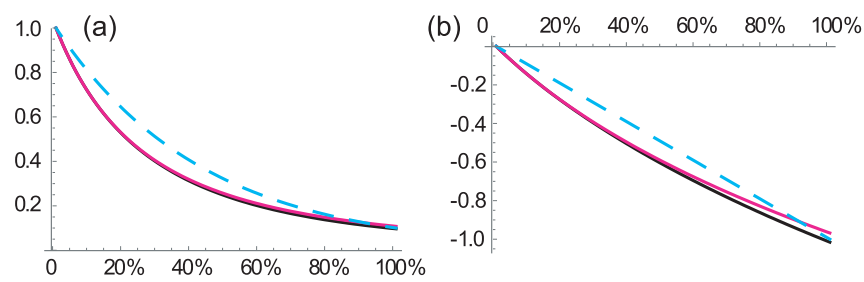

FIG. 12. (a) Fidelity $F(|\phi\rangle,|\psi\rangle)$ is plotted in black curve. Norm of the output from the quantum gate with resistance is plotted in magenta. Cyan dotted curves represent an approximate relation $10^{-R}$. The horizontal axis is the strength of resistance. (b) Corresponding $\log _{10}$ plot. 
merits. First, the speed of computations is independent of the number of wires, since the Kirchhof law automatically performs computations. It is similar to the original idea from Feynman [1] about quantum computations, where the Schrödinger dynamics automatically performs computations. Thus, the speed is contrasted with that of classical computers. Second, our scheme performs any quantum algorithms based on electric circuits although we need a large number of elements for a large-scale quantum computation.

We compare our quantum-walk scheme with the original proposal from Childs [6]. In electric circuits, only subdivided graphs are realized by the way of rewriting the Kirchhof law in the form of the Schrödinger equation. In particular, the original mixing gate proposed by Childs is unrealizable in electric circuits. Instead, we have proposed a similar but different widget. In this sense, our findings are nontrivial although the action of universal quantum gates is identical.

When we use inductors of the order of $1 \mathrm{nH}$ and capacitors of the order of $1 \mathrm{pF}$, the resonant frequency exceeds $1 \mathrm{GHz}$.
Then, we expect high-speed computations. We have pointed out that the fidelity remains almost perfect provided we can design some compensation circuits to recover the normalization electrically. Our results will open a way to simulate any quantum algorithms based on electric circuits. Merits are that we may control them by classical computers seamlessly integrated and that they work at room temperature. We hope to implement scalability in future works by including internal degrees of freedom to quantum walkers in electric circuits.

\section{ACKNOWLEDGMENTS}

The author is very much grateful to E. Saito and N. Nagaosa for helpful discussions on the subject. This work is supported by the Grants-in-Aid for Scientific Research from MEXT KAKENHI (Grants No. JP17K05490, No. JP15H05854, and No. JP18H03676). This work is also supported by CREST, JST (No. JPMJCR16F1).
[1] R. Feynman, Int. J. Theor. Phys. 21, 467 (1982).

[2] D. P. DiVincenzo, Science 270, 255 (1995).

[3] D. Deutsch, Proc. R. Soc. A. 400, 97 (1985)

[4] C. M. Dawson and M. A. Nielsen, arXiv:quant-ph/0505030.

[5] M. Nielsen and I. Chuang, Quantum Computation and Quantum Information (Cambridge University Press, Cambridge, 2016), p. 189.

[6] A. M. Childs, Phys. Rev. Lett. 102, 180501 (2009).

[7] M. Varbanov and T. A. Brun, Phys. Rev. A 80, 052330 (2009).

[8] Benjamin A. Blumer, M. S. Underwood, and D. L. Feder, Phys. Rev. A 84, 062302 (2011).

[9] A. P. Hines and P. C. E. Stamp, Phys. Rev. A 75, 062321 (2007).

[10] N. B. Lovett, S. Cooper, M. Everitt, M. Trevers, and V. Kendon, Phys. Rev. A. 81, 042330 (2010).

[11] A. M. Childs, D. Gosset, and Z. Webb, Science 339, 791 (2013).

[12] M. S. Underwood and D. L. Feder, Phys. Rev. A 82, 042304 (2010).

[13] M. S. Underwood and D. L. Feder, Phys. Rev. A 85, 052314 (2012).

[14] D. Solenov, Quantum Info. Comput. 17, 415 (2017).

[15] Y. L. Gregory, R. Steinbrecher, A. D. Bookatz, and D. Englund, npj Quantum Inf. 4, 2 (2018).

[16] A. Peruzzo, M. Lobino, J. C. F. Matthews, N. Matsuda, A. Politi, K. Poulios, X.-Q. Zhou, Y. Lahini, N. Ismail, K. Wörhoff, Y. Bromberg, Y. Silberberg, M. G. Thompson, and J. L. OBrien, Science 329, 1500 (2010).

[17] Z. Yan, Y.-R. Zhang, M. Gong, Y. Wu, Y. Zheng, S. Li, C. Wang, F. Liang, J. Lin, Y. Xu, C. Guo, L. Sun, C.-Z. Peng, K. Xia, H. Deng, H. Rong, J. Q. You, F. Nori, H. Fan, X. Zhu, and J.-W. Pan, Science 364, 753 (2019).

[18] M. Karski, L. Förster, J.-M. Choi, A. Steffen, W. Alt, D. Meschede, and A. Widera, Science 325, 174 (2009).

[19] P. M. Preiss, R. Ma, M. E. Tai, A. Lukin, M. Rispoli, P. Zupancic, Y. Lahini, R. Islam, and M. Greiner, Science 347, 1229 (2015)
[20] C. H. Lee, S. Imhof, C. Berger, F. Bayer, J. Brehm, L. W. Molenkamp, and T. Kiessling, and R. Thomale, Commun. Phys. 1, 39 (2018).

[21] S. Imhof, C. Berger, F. Bayer, J. Brehm, L. Molenkamp, T. Kiessling, F. Schindler, C. H. Lee, M. Greiter, T. Neupert, and R. Thomale, Nat. Phys. 14, 925 (2018).

[22] M. Serra-Garcia, R. Susstrunk, and S. D. Huber, Phys. Rev. B 99, 020304(R) (2019).

[23] T. Helbig, T. Hofmann, C. H. Lee, R. Thomale, S. Imhof, L. W. Molenkamp, and T. Kiessling, Phys. Rev. B 99, 161114(R) (2019).

[24] E. I. Rosenthal, N. K. Ehrlich, M. S. Rudner, A. P. Higginbotham, and K. W. Lehnert, Phys. Rev. B 97, 220301(R) (2018).

[25] Y. Lu, N. Jia, L. Su, C. Owens, G. Juzeliunas, D. I. Schuster, and J. Simon, Phys. Rev. B 99, 020302(R) (2019).

[26] M. Ezawa, Phys. Rev. B 98, 201402(R) (2018).

[27] T. Hofmann, T. Helbig, C. H. Lee, M. Greiter, and R. Thomale, Phys. Rev. Lett. 122, 247702 (2019).

[28] K. Luo, R. Yu, and H. Weng, Research 2018, 6793752 (2018).

[29] M. Ezawa, Phys. Rev. B 99, 201411(R) (2019).

[30] M. Ezawa, Phys. Rev. B 100, 045407 (2019).

[31] T. Helbig, T. Hofmann, S. Imhof, M. Abdelghany, T. Kiessling, L. W. Molenkamp, C. H. Lee, A. Szameit, M. Greiter, and R. Thomale, arXiv:1907.11562.

[32] M. Ezawa, arXiv:1907.06911.

[33] M. Ezawa, Phys. Rev. B 100, 165419 (2019).

[34] V. Vedral, A. Barenco, and A. Ekert, Phys. Rev. A 54, 147 (1996).

[35] D. Coppersmith, Techn. Rep. RC19642, IBM (1994).

[36] M. Nielsen and I. Chuang, Quantum Computation and Quantum Information (Cambridge University Press, Cambridge, 2000). 